\title{
Medullary colonic carcinoma with microsatellite instability has lower survival compared with conventional colonic adenocarcinoma with microsatellite instability
} \author{
Erika B. Ruiz-García ${ }^{3}$, Horacio N. López-Basave ${ }^{1}$, German Calderillo-Ruiz ${ }^{3}$, José M. Aguilar-Romero ${ }^{4}$, \\ Jorge A. Domínguez-Rodríguez ${ }^{4}$, Ángel Herrera-Gómez ${ }^{1}$, Abelardo Meneses-García² \\ ${ }^{1}$ Surgical Oncology, Instituto Nacional de Cancerología, México City, Mexico \\ 2Department of Anatomic Pathology, Instituto Nacional de Cancerología, México City, Mexico \\ ${ }^{3}$ Medical Oncology, Instituto Nacional de Cancerología, México City, Mexico \\ ${ }^{4}$ Medical School, Universidad Nacional Autónoma de México (UNAM), Mexico City, Mexico
}

Miguel A. Gómez-Álvarez ${ }^{1}$, Leonardo S. Lino-Silva², Rosa A. Salcedo-Hernández ${ }^{1}$, Alejandro Padilla-Rosciano ${ }^{1}$,

Gastroenterology Rev 2017; 12 (3): 208-214

DOI: $10.5114 / p g .2016 .64740$

Key words: colorectal, cancer, microsatellite instability, medullary carcinoma, survival.

Address for correspondence: Leonardo S. Lino-Silva MD, Department of Anatomic Pathology, Instituto Nacional de Cancerología, Av. San Fernando 22 Col. Sección XVI, CP. 14080, México City, México, phone: +52 55 3426-5921, e-mail: saul.lino.sil@gmail.com

\begin{abstract}
Introduction: Colorectal medullary carcinoma $(M C)$ is a rare subtype of poorly differentiated adenocarcinoma (PDA) with unclear prognostic significance. Microsatellite instable (MSI) colorectal carcinomas have demonstrated better prognosis in clinical stage II.

Aim: To analyze the survival and clinicopathological characteristics of MCs versus PDAs with MSI in clinical stage III.

Material and methods: We studied 22 cases of PDAs with MSI versus 10 MCs.

Results: Of the $10 \mathrm{MCs}, 7$ patients were men; the mean age was $57.8 \pm 5.6$ years. The mean tumor size was $9.6 \pm 4.1 \mathrm{~cm}$, and the primary site was the right colon in 9; 7 patients showed lymph node metastases (LNM) and lymphovascular invasion (LVI). Of the 22 PDA cases, 12 (54.5\%) were women with a mean age of $75 \pm 16.1$ years. The mean tumor size was $6.4 \pm 3.2 \mathrm{~cm}$. Twelve (54.5\%) presented in the right colon, 21 (95.5\%) showed LNM and 7 (31.8\%) LVI. Follow-up was $32 \pm 8$ months, with a 5-year overall survival of $42.9 \%$ for MCs and $76.6 \%$ for PDAs $(p=0.048)$. Univariate analysis found local recurrence $(p=0.001)$ and medullary subtype $(p=0.043)$ associated with lower survival.

Conclusions: Medullary carcinomas were of greater tumor size and associated with more LVI and worse survival versus PDAs with MSI in stage III.
\end{abstract}

\section{Introduction}

Colorectal medullary carcinoma $(M C)$ is a distinctive, poorly differentiated adenocarcinoma (PDA) increasingly recognized and studied in the last two decades. Formerly known as 'large cell adenocarcinoma with minimal differentiation', it is now being referred to as 'medullary' carcinoma because of its organoid architecture that is similar to the pattern seen in developing embryonic organs [1].

However, it is important not to use this term in the colorectal literature with the same connotation as it is used in breast and thyroid, because the clinicopathologi- cal features are quite different. Medullary carcinoma has been included as a distinct histological type in the World Health Organization (WHO) classification of colorectal carcinomas, wherein it is described as being characterized by sheets of malignant cells with vesicular nuclei, and prominent nucleoli, along with prominent intraepithelial lymphocytic infiltrate [2]. The MC is a solid variety of adenocarcinoma with very little glandular differentiation. Although they are morphologically similar to undifferentiated adenocarcinoma (UDA), they tend to display distinct clinical behavior: they are typically more common in older females, less likely to present with nodal involvement and 
generally have a better prognosis [3]. However, there are very few data published in this respect. Another characteristic feature of $\mathrm{MC}$ is its strong association with microsatellite instability (MSI) in at least $60 \%$ of cases $[4,5]$.

Most colorectal carcinomas are adenocarcinomas of conventional type (adenocarcinoma NOS, not otherwise specified). Adenocarcinomas are graded into well-differentiated, moderately differentiated, poorly differentiated and undifferentiated tumors (grades 1, 2, 3 and 4 , respectively) depending on the proportion of gland formation in the least differentiated component of the tumor away from the invasive edge, according to the WHO criteria. This is a subjective assessment with low levels of agreement among pathologists [6], but histologic grading has been shown to be an independent prognostic factor for colorectal carcinoma, particularly true for the poorly differentiated subgroup [7-10]. The WHO and the American Joint Committee on Cancer (AJCC) recommend a 2-tiered histologic grading system: low grade for well-differentiated and moderately differentiated adenocarcinomas (50-100\% gland formation) and high grade for poorly differentiated adenocarcinomas (0-49\% gland formation) and undifferentiated adenocarcinoma (0-1\% gland formation) [11]. Testing tumors for microsatellite instability (MSI) by immunohistochemistry for mismatch repair (MMR) proteins MLH1, MSH2, PMS2, and MSH6 and/or by molecular based methods is routinely performed for patients diagnosed with colorectal carcinoma, primarily to screen for Lynch syndrome and because of it has been reported to be a strong positive prognostic factor [12-15]. Up to $15 \%$ of all colorectal carcinomas demonstrate MSI; the rest are called microsatellite stable (MSS) carcinomas. Some histologic subtypes of colorectal carcinomas are more commonly observed in MSI tumors, including medullary carcinomas, mucinous adenocarcinomas, and

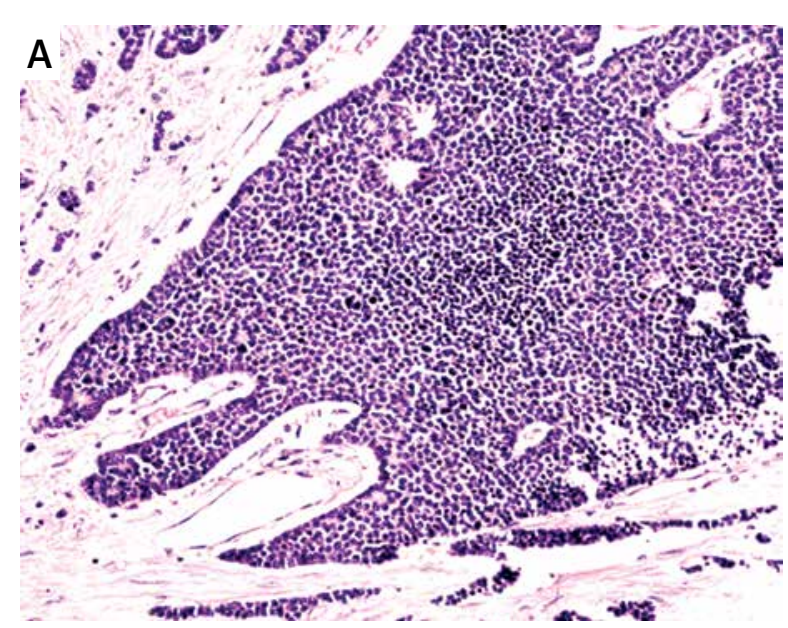

signet ring cell carcinomas [16]. The adverse prognosis associated with the poor differentiation of most of these tumor subtypes contrasts with the positive prognosis associated with MSI. Consequently, the current WHO histologic grading does not apply to colorectal MC.

\section{Aim}

According to the few published series and data about survival and prognosis of this tumor, and to further characterize the clinicopathologic attributes and survival of MCs, the present study compares the morphological and behavioral features of MCs with MSI with those of poorly differentiated cases of conventional adenocarcinomas (PDA) matched for clinical stage (AJCC stage III) and MSI.

\section{Material and methods}

We searched in our institutional surgical pathology files from January 2005 to December 2013 to retrieve all cases of CRC that had been coded as "undifferentiated", "poorly differentiated", "anaplastic", "medullary", and "lymphoepithelial carcinoma". We reviewed all the $\mathrm{H}+$ E-stained sections of each lesion obtained thought to obtain the carcinomas that meet the criteria for medullary and poorly differentiated adenocarcinomas. Carcinomas that met the criteria were retained for further study. Only resection specimens were included in the analysis.

The morphologic features of each of the 2 study groups were as follows: MCs were required to demonstrate sheets, nests, and trabeculae of large polygonal cells with high nuclear/cytoplasmic (N/C) ratios and amphophilic cytoplasm, with variably interspersed mature lymphocytes (Figure 1 A). Nuclei were vesicular, with prominent nucleoli (Figure 1 B). Focal cytoplasmic vacuolization and a minor admixture of a conventional

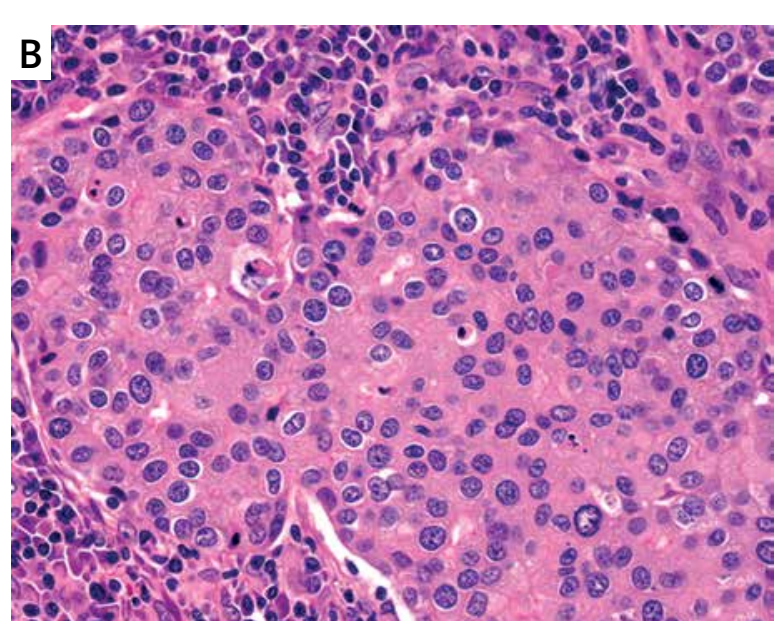

Figure 1. Morphologic characteristics of medullary carcinoma. Architectural solid nest of neoplastic cells (A) with round nuclei with coarse chromatin (B) 
adenocarcinoma component were allowed in such lesions. Neuroendocrine differentiation of MCs was ruled out at the time of the diagnosis.

Characteristics of PDA included rudimentary but definite gland formation, infiltrative growth, and basal orientation of nuclei within tumor glands, vesicular chromatin, discernible nucleoli, and foci of dirty geographic necrosis. The age and sex of each patient in the study group, the anatomic locations of the tumors, their stages (according to American Joint Committee on Cancer definitions) and follow-up information were recorded for all cases. Only cases of nonhereditary colorectal neoplasia were accepted into the final study group, and tumors arising in the context of inflammatory bowel disease also were excluded.

Paraffin sections of each case chosen for study were cut at $4 \mu \mathrm{m}$, mounted on adhesive slides, and rehydrated in a standard manner for immunohistochemical analysis. They were subjected to microwave-mediated epitope retrieval after immersion in citrate buffer and labeled with antibodies to MSH2, MSH6, MLH1, PMS2, PML1 and P53 (Table I).

Avidin-biotin-peroxidase complex immunodetection ensued, with development of chromogenic precipitates in a solution of diaminobenzidine hydrochloride $(0.25$ $\mathrm{mg} / \mathrm{ml}$ ). Slides were counterstained with hematoxylin and examined by light microscopy. Immunostaining was scored as positive if the tumor cells demonstrated labeling for the determinants in question. The tumors were considered with MSI when the expression of at least one of the following markers was lost: MSH2, MSH6, MLH1, PMS2, PML1. All cases with the expression of all markers and P53 were classified as MSS carcinomas.

After classifying the PDAs by immunohistochemistry, we selected only those carcinomas with MSI and clinical stage IIIA and IIIB according to the AJCC staging system [12], obtaining the final study population of $10 \mathrm{MCs}$ and 22 PDAs.

\section{Statistical analysis}

Data were analyzed using the Statistical Package for the Social Sciences statistical analysis software (version
12.0, SPSS, Inc., Chicago, IL), using the $\chi^{2}$ method and log rank test. Results were considered significant at a $p$-value of $\leq 0.05$.

\section{Results}

\section{Clinicopatholologic findings}

The clinical characteristics of the study population (32 cases) of MSI carcinomas are summarized in Table II. Of a total of 10 MCs, 7 (70\%) patients were men and only 3 patients were women; the mean age was 57.8 \pm 5.6 years (range: $28-74$ years), the mean tumor size was $9.6 \pm 4.1 \mathrm{~cm}$ (range: $5-18 \mathrm{~cm}$ ), the carcinoembryonic antigen (CEA) was elevated in 5 (50\%) patients, the primary site of the tumor was the right colon in $9(90 \%)$ cases and the transverse colon in the remaining case. The main tumor burden infiltrated into subserosal adipose tissue in 5 (50\%) cases, muscularis propria in $2(20 \%)$ cases and serosa in 3 cases. Seven patients showed lymph node metastases and lymphovascular invasion. No case showed perineural invasion. All cases were treated with surgery as initial treatment with complete resection (clear margins, R0) was achieved in 9 patients and in one microscopic tumor in the surgical margin was identified (R1 resection). Six (60\%) patients received adjuvant chemotherapy based on capecitabine plus oxaliplatin with an average of 6 cycles.

For the PDA group 22 cases were analyzed. Ten (45.5\%) patients were men and 12 (54.5\%) patients were women; the mean age was $75 \pm 16.1$ years (range: 24-79 years), the mean tumor size was $6.4 \pm 3.2 \mathrm{~cm}$ (range: $2-12 \mathrm{~cm}$ ), the CEA was elevated in 10 (4.5\%) patients, the primary site of the tumor was the right colon in 12 (54.5\%) cases and the transverse/descendent colon in the remaining 10 (45.5\%) cases. The main tumor burden infiltrated into subserosal adipose tissue in 15 (68.2\%) cases, serosa in $3(13.6 \%)$ cases and adjacent tissues in 4 (18.2\%) cases. Twenty-one (95.5\%) patients showed lymph node metastases and 7 (31.8\%) lymphovascular invasion. Four (18.2\%) cases showed venous invasion and 2 (9.1\%) cases perineural invasion. All cases were treated with surgery as initial treatment with complete resection achieved in 19 (86.4\%) patients and

Table I. Antibodies used in the immunohistochemical studies

\begin{tabular}{llcr} 
Antibody (Clone) & Source & Dilution & Antigen retrieval \\
\hline MLH1 (ES05) & Dako, Carpintera, CA & $1: 50$ & Heat (EDTA), 15 min \\
\hline MSH2 (FE11) & Dako, Carpintera, CA & $1: 50$ & Heat (EDTA), 15 min \\
\hline MSH6 (EP49) & Dako, Carpintera, CA & $1: 50$ & Heat (EDTA), 15 min \\
\hline PSM2 (EP51) & Dako, Carpintera, CA & $1: 40$ & Heat (EDTA), 15 min
\end{tabular}


Table II. Clinicopathologic characteristics of medullary carcinomas compared with conventional adenocarcinomas

\begin{tabular}{|c|c|c|c|}
\hline Parameter & $\begin{array}{c}\text { Medullary } \\
\text { carcinoma, } \\
n(\%)\end{array}$ & $\begin{array}{l}\text { Conventional } \\
\text { adenocarci- } \\
\text { noma, } n(\%)\end{array}$ & $P$-value \\
\hline \multicolumn{3}{|l|}{ Gender: } & \multirow[t]{3}{*}{0.409} \\
\hline Female & $3(30)$ & $10(45.5)$ & \\
\hline Male & $7(70)$ & $12(54.5)$ & \\
\hline \multicolumn{3}{|l|}{ CEA seric level: } & \multirow[t]{3}{*}{0.811} \\
\hline Normal & $5(50)$ & $12(54.5)$ & \\
\hline Elevated & $5(50)$ & $10(45.5)$ & \\
\hline \multicolumn{3}{|l|}{ Site: } & \multirow[t]{3}{*}{0.050} \\
\hline Right colon & $9(90)$ & $12(54.5)$ & \\
\hline Transverse/left colon & $1(10)$ & $10(45.5)$ & \\
\hline \multicolumn{3}{|l|}{ Tumor invasion: } & \multirow[t]{5}{*}{0.056} \\
\hline pT2 & $2(20)$ & 0 & \\
\hline pT3 & $5(50)$ & $15(68.2)$ & \\
\hline рT4a & $3(30)$ & $3(13.6)$ & \\
\hline pT4b & 0 & $4(18.2)$ & \\
\hline \multicolumn{3}{|l|}{ Lymph node stage: } & \multirow[t]{6}{*}{0.160} \\
\hline pNO & $3(30)$ & $1(4.5)$ & \\
\hline pN1a & $1(10)$ & $5(22.7)$ & \\
\hline $\mathrm{pN1b}$ & $4(40)$ & $7(31.8)$ & \\
\hline pN2a & 0 & $5(22.7)$ & \\
\hline $\mathrm{pN} 2 \mathrm{~b}$ & $2(20)$ & $4(18.2)$ & \\
\hline \multicolumn{3}{|l|}{ Lymph node metastasis: } & \multirow[t]{3}{*}{0.044} \\
\hline No & $3(30)$ & $1(4.5)$ & \\
\hline Yes & $7(70)$ & $21(95.5)$ & \\
\hline \multicolumn{3}{|l|}{ Lymphovascular invasion: } & \multirow[t]{3}{*}{0.044} \\
\hline No & $3(30)$ & $15(68.2)$ & \\
\hline Yes & 7 (70) & 7 (31.8) & \\
\hline
\end{tabular}

\begin{tabular}{|c|c|c|c|}
\hline Parameter & $\begin{array}{c}\text { Medullary } \\
\text { carcinoma, } \\
n(\%)\end{array}$ & $\begin{array}{l}\text { Conventional } \\
\text { adenocarci- } \\
\text { noma, } n(\%)\end{array}$ & $P$-value \\
\hline Vascular invasion: & & & 0.354 \\
\hline No & $10(100)$ & $18(81.8)$ & \\
\hline Yes & 0 & $4(18.2)$ & \\
\hline Perineural invasión: & & & 0.325 \\
\hline No & $10(100)$ & $20(90.9)$ & \\
\hline Yes & 0 & $2(9.1)$ & \\
\hline Resection: & & & 0.777 \\
\hline RO & $9(90)$ & $19(86.4)$ & \\
\hline $\mathrm{R} 1$ & $1(10)$ & $3(13.6)$ & \\
\hline Recurrence: & & & 0.660 \\
\hline No & $8(80)$ & $16(72.7)$ & \\
\hline Yes & $2(20)$ & $6(27.3)$ & \\
\hline Adjuvant chemotherapy & & & 0.094 \\
\hline No & $4(40)$ & $3(13.6)$ & \\
\hline Yes & $6(60)$ & $19(86.4)$ & \\
\hline Progression: & & & 0.488 \\
\hline No & $7(70)$ & $17(77.3)$ & \\
\hline Yes & $3(30)$ & $5(22.7)$ & \\
\hline Death: & & & 0.660 \\
\hline No & $7(70)$ & $17(77.3)$ & \\
\hline Yes & $3(30)$ & $5(22.7)$ & \\
\hline $\begin{array}{l}\text { 5-year overall survival } \\
(\%)\end{array}$ & 42.9 & 76.6 & 0.048 \\
\hline Age [years] & $57.8 \pm 5.6$ & $75 \pm 16.1$ & 0.074 \\
\hline Tumor size $[\mathrm{cm}]$ & $9.6 \pm 4.1$ & $6.4 \pm 3.2$ & 0.035 \\
\hline
\end{tabular}

CEA - carcinoembrionic antigen.

in $3(13.6 \%)$ cases microscopic tumor in the surgical margin was identified (R1 resection). Nineteen (86.4\%) patients received adjuvant chemotherapy based on 5 -fluorouracil with an average of 6 cycles.

\section{Outcome and survival analysis}

The average follow-up time was $32 \pm 8$ months (range: $15-38$ months). For the MC group during the follow-up, 2 patients showed recurrence and 3 cases showed disease progression with lung parenchymal metastasis. For the PDA group 6 (27.3\%) patients showed recurrence and 5 (22.7\%) progression. None of these differences were statistically significant.

In the survival analysis, 3 (30\%) patients died of disease in the MC group, with a 5 -year overall survival (OS) of $42.9 \%$. For the PDA group, 5 patients died of

the disease $(22.7 \%)$, with a 5 -year OS of $76.6 \%$, with a statistically significant difference compared with MCs ( $p=0.048$, Figure 2).

Univariate survival analysis (log-rank test) found that factors associated with poor prognosis were recurrence ( $p=0.001$, Figure 3 ) and medullary subtype $(p=0.043$, Table III). In the multivariate analysis none of the analyzed factors showed an association with worse survival.

\section{Discussion}

During the past 25 years, several distinct morphologic variants of colorectal carcinoma (CRC) have been identified, including mucinous carcinoma, signet-ring cell adenocarcinoma, large-cell carcinoma, neuroendocrine carcinoma, "amphicrine" carcinoma, sarcomatoid 


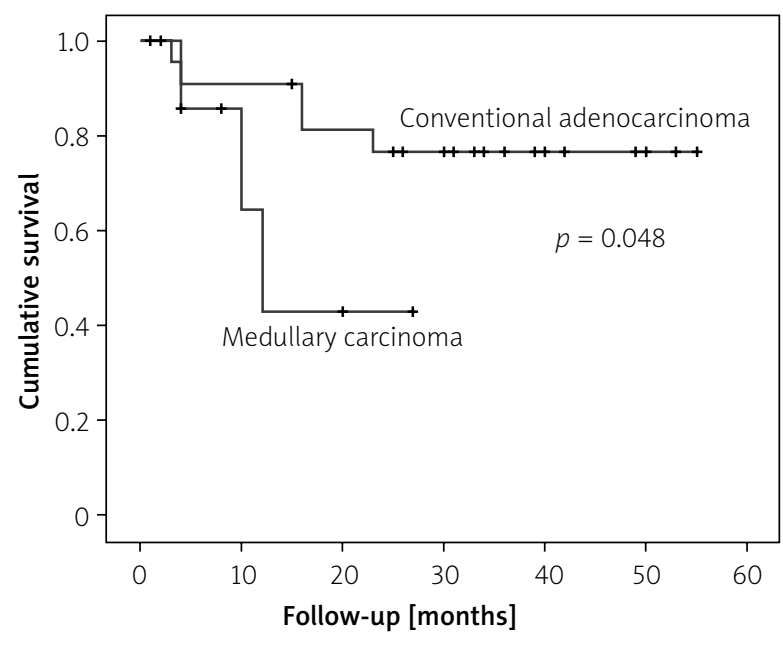

Figure 2. Overall survival comparison between medullary carcinomas and poorly differentiated carcinomas

Table III. Univariate survival analysis

\begin{tabular}{lccc} 
Variable & $\chi^{2}$ value & $\boldsymbol{P}$-value & $\begin{array}{c}\text { Confident } \\
\text { interval }\end{array}$ \\
\hline Recurrence & 11.629 & 0.001 & $4.68-15.35$ \\
\hline Medullary type & 3.404 & 0.043 & $2.5-4.21$ \\
\hline Female sex & 3.176 & 0.075 & $1.5-3.0$ \\
\hline Right colon location & 3.0629 & 0.057 & $1.05-2.58$ \\
\hline Lymph node metastasis & 0.109 & 0.741 & $0.58-1.36$ \\
\hline CEA level (high) & 0.788 & 0.375 & $0.53-1.02$
\end{tabular}

carcinoma ("carcinosarcoma"), squamous cell carcinoma, lymphoepithelioma-like carcinoma, and "medullary" carcinoma. Aside from having clinical evolutions that are potentially different from those of conventional forms of colorectal carcinoma, these other morphologic subtypes also evoke differential diagnoses and raise various questions about correlations between their histologic images and basic cellular properties.

Medullary carcinoma of the colon is a relatively recent addition to the histological types of colorectal carcinomas and has been dealt with in only a handful of studies that have primarily dealt with its pathological aspects. As early as 1977, Gibbs reported a small series of undifferentiated adenocarcinomas with a tendency to grow to larger sizes before producing symptoms but had a favorable prognosis [17]. In 1997, Ruschoff et al. reported a series of poorly differentiated non-glandular colorectal adenocarcinomas, most of which exhibited an expansive growth pattern and significant peritumoral lymphoid infiltrate, resembling solid or medullary carcinomas of the stomach [18]. They also noted that they

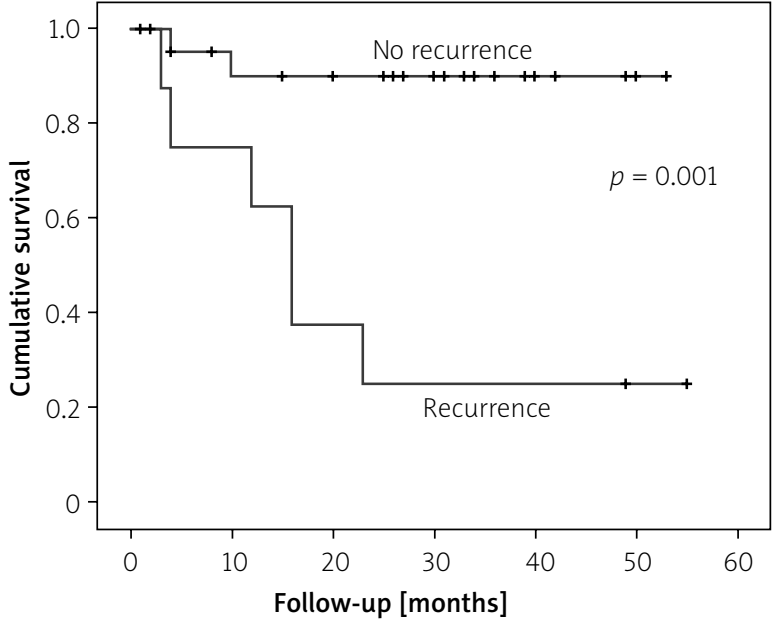

Figure 3. Survival curves for recurrence (KaplanMeier method)

had high MSI and generally showed good prognosis. In 1999, Jessurun et al. described a series of 11 cases of colonic adenocarcinoma that displayed a predominantly solid, non-glandular component and were negative for neuroendocrine markers [19]. All patients were women with tumors in the cecum or proximal colon. The authors concluded that this non-glandular adenocarcinoma should be distinguished from the other more aggressive non-glandular adenocarcinomas. Lanza et al. identified 45 MCs among 1,265 surgical specimens over a period of 10 years [3]. They observed that these tumors were typically diploid, p53-negative, demonstrated widespread MSI, and had a favorable prognosis compared to well-differentiated and PDAs. Following this, a few other studies confirmed the high frequency of MSI, loss of p53 and CDX2 expression and favorable prognosis of MCs compared to PDA and neuroendocrine tumors [20]. A recent interesting observation was made by Winn, who noted that although MCs and PDA may be difficult to differentiate by light microscopy, MCs frequently stained positive for MUC-1 and MUC-2, indicating that MCs still retain intestinal differentiation [21]. They also showed they can be differentiated from other undifferentiated carcinomas by strong calretinin staining and loss of MLH-1 and CDX2 staining.

Our study of 10 MCs pointed out new findings as well in this carcinoma. In our study group the predominant sex group was men, the tumors showed a very high proportion of lymphovascular invasion (70\%), tumor size was greater $(9.6 \mathrm{~cm}$ vs. $6.4 \mathrm{~cm})$, the age of presentation was lower ( 57.8 years vs. 75 years), there was absence of perineural invasion, all cases showed MSI and, more important, compared with PDA with MSI matched by AJCC stage, MCs showed lower survival, data contrary to most published series. The explana- 
tion of these findings it is not clear. Although most published series claimed that medullary carcinoma has better prognosis, the definition and identification of MCs are not very clearly stated. However, the better prognosis of MSI carcinomas is very debatable; there are many studies demonstrating that MSI have at least similar clinical behavior compared with MSS carcinomas, and the clinical benefit has been observed only in stage II tumors [22-27]. This heterogeneity of clinical behavior could be explained by the multitude of molecular heterogeneity underlying MSI tumors, because they also harbor BRAF mutations and KRAS mutations. We believe that MSI does not have strong prognostic significance, and our data confirm this affirmation. We consider that histologic grade could have the most prognosis significance because by definition all MCs are grade 3 or 4 carcinomas, and possibly this could explain their worse prognosis in our study. Another interesting finding is the fact that high lymphovascular invasion did not correlate with the $\mathrm{N}$ stage; we do not have a good explanation for that. Tumor burden could explain some of these findings; however, tumor size has not been demonstrated to be a poor prognostic factor [28].

\section{Conclusions}

Medullary carcinomas of the colorectum are a rare subset of minimally differentiated adenocarcinomas, which were more common in men. They are more common by far in the proximal colon, with almost no occurrences in the left colon, suggesting a possible role of site-specific variations such as colonic flora, intraluminal nutrient content and composition. All MCs of our series were MSI. MCs commonly presented in stage III, were of higher tumor size and were associated with more lymphovascular invasion and with worse survival compared with stage III PDAs with MSI. However, further studies are necessary, including molecular analysis with regard to the specific subsets of MCs. Finally, the authors suspect that medullary carcinoma of the colon may be increasingly identified hereafter, possibly revealing new behavior patterns identifiable in the future.

\section{Conflict of interest}

The authors declare no conflict of interest.

\section{References}

1. Wick MR, Vitsky JL, Ritter JH, et al. Sporadic medullary carcinoma of the colon: a clinicopathologic comparison with nonhereditary poorly differentiated enteric-type adenocarcinoma and neuroendocrine colorectal carcinoma. Am J Clin Pathol 2005; 123: 56-65.

2. Hamilton SR, Aaltonen LA (eds). Pathology and genetics of tumors of the digestive system. World Health Organization Classification of Tumors. IARC Press, Lyon 2000.
3. Lanza G, Gafa R, Matteuzzi M, Santini A. Medullary-type poorly differentiated adenocarcinoma of the large bowel: a distinct clinicopathologic entity characterized by microsatellite instability and improved survival. J Clin Oncol 1999; 17: 2429-38.

4. Alexander J, Watanabe T, Wu TT, et al. Histopathological identification of colon cancer with microsatellite instability. Am J Pathol 2001; 158: 527-35.

5. Hinoi T, Tani M, Lucas PC, et al. Loss of CDX2 expression and microsatellite instability are prominent features of large cell minimally differentiated carcinomas of the colon. Am J Pathol 2001; 159: 2239-48.

6. Chandler I, Houlston RS. Interobserver agreement in grading of colorectal cancers - findings from a nationwide web-based survey of histopathologists. Histopathology 2008; 52: 494-9.

7. Thomas GD, Dixon MF, Smeeton NC, Williams NS. Observer variation in the histological grading of rectal carcinoma. J Clin Pathol 1983; 36: 385-91.

8. Chapuis PH, Dent OF, Fisher R, et al. A multivariate analysis of clinical and pathological variables in prognosis after resection of large bowel cancer. Br J Surg 1985; 72: 698-702.

9. Compton CC, Fielding LP, Burgart LJ, et al. Prognostic factors in colorectal cancer. College of American Pathologists Consensus Statement 1999. Arch Pathol Lab Med 2000; 124: 979-94.

10. Halvorsen TB, Seim E. Degree of differentiation in colorectal adenocarcinomas: a multivariate analysis of the influence on survival. J Clin Pathol 1988; 41: 532-7.

11. Jass JR, Atkin WS, Cuzick J, et al. The grading of rectal cancer: historical perspectives and a multivariate analysis of 447 cases. Histopathology 1986; 10: 437-59.

12. Edge SB, Byrd DR, Compton CC, et al. (eds.). American Joint Committee on Cancer Cancer Staging Manual. Springer, Chicago 2010.

13. Benatti P, Gafa R, Barana D, et al. Microsatellite instability and colorectal cancer prognosis. Clin Cancer Res 2005; 11: 8332-40.

14. Alexander J, Watanabe T, Wu TT, et al. Histopathological identification of colon cancer with microsatellite instability. Am J Pathol 2001; 158: 527-35.

15. Sinicrope FA, Rego RL, Halling KC, et al. Prognostic impact of microsatellite instability and DNA ploidy in human colon carcinoma patients. Gastroenterology 2006; 131: 729-37.

16. Shia J, Ellis NA, Paty PB, et al. Value of histopathology in predicting microsatellite instability in hereditary nonpolyposis colorectal cancer and sporadic colorectal cancer. Am J Surg Pathol 2003; 27: 1407-17.

17. Gibbs NM. Undifferentiated carcinoma of the large intestine. Histopathology 1977; 1: 77-84.

18. Ruschoff J, Dietmaier W, Luttges J, et al. Poorly differentiated colonic adenocarcinoma, medullary type: clinical, phenotypic, and molecular characteristics. Am J Pathol 1997; 150: 1815-25.

19. Jessurun J, Romero-Guadarrama M, Manivel JC. Medullary adenocarcinoma of the colon: clinicopathologic study of 11 cases. Hum Pathol 1999; 30: 843-8.

20. Winn B, Tavares R, Fanion J, et al. Differentiating the undifferentiated: immunohistochemical profile of medullary carcinoma of the colon with an emphasis on intestinal differentiation. Hum Pathol 2009; 40: 398-404.

21. Thirunavukarasu P, Sathaiah $M$, Singla $S$, et al. Medullary carcinoma of the large intestine: a population based analysis. Int J Oncol 2010; 37: 901-7. 
22. Phipps Al, Limburg PJ, Baron JA. Association between molecular subtypes of colorectal cancer and patient survival. Gastroenterology 2015; 148: 77-87.e2.

23. Saridaki Z, Souglakos J, Georgoulias V. Prognostic and predictive significance of MSI in stages II/III colon cancer. World J Gastroenterol 2014; 20: 6809-14.

24. Kim JH, Kang GH. Molecular and prognostic heterogeneity of microsatellite-unstable colorectal cancer. World J Gastroenterol 2014; 20: 4230-43.

25. Hveem TS, Merok MA, Pretorius ME, et al. Prognostic impact of genomic instability in colorectal cancer. Br J Cancer 2014; 110: 2159-64.

26. Setaffy L, Langner C. Microsatellite instability in colorectal cancer: clinicopathological significance. Pol J Pathol 2015; 66: 203-18.

27. Forner A, Díaz-González A, Liccioni A, Vilana R. Prognosis prediction and staging. Best Pract Res Clin Gastroenterol 2014; 28: 855-65.

28. Abe J, Urano T, Konno $\mathrm{H}$, et al. Larger and more invasive colorectal carcinoma contains larger amounts of plasminogen activator inhibitor type 1 and its relative ratio over urokinase receptor correlates well with tumor size. Cancer 1999; 86: 2602-11.

Received: 1.07.2015

Accepted: 1.02 .2016 